interesting to determine whether this confidence will be maintained at three months and one year.

It is hoped that improved staff confidence scores will be achieved and maintained with resultant increased use of the handheld fan and improved management of the symptom of breathlessness.

Conclusion It is hoped that the anticipated results will demonstrate improved confidence and improved management of the symptom of breathlessness. Future plans will be centred on making the training available in other care settings and teams with resultant up-skilling of the workforce in non-pharmacological management of breathlessness in palliative care.

\section{P-113 EVALUATION OF A FATIGUE AND BREATHLESSNESS PROGRAMME BY HOSPICE ISLE OF MAN}

Alison Christian, Jo Beard, Sarah M McGhee. Hospice Isle of Man, Strang, Isle of Man

\subsection{6/bmjspcare-2018-hospiceabs.138}

Background A programme was developed at Hospice Isle of Man to provide support for people who are experiencing extreme tiredness and/or breathlessness. The programme involves exercise, relaxation and education.

Aim To evaluate the programme using validated outcome measures and the views and experience of the participants.

Methods We used the Self-reported Chronic Respiratory Disease Questionnaire (Chauvin, Rupley, Meyers et al., 2008) which is a quality of life measure designed for those with respiratory problems. It asks individuals to rate their current state on four dimensions: dyspnoea, fatigue, emotional function and mastery (feeling of control over disease) using a seven point scale. This is done for their five most important activities in which they have been limited by symptoms, giving a total score for each dimension. Previous validations (Chauvin, Rupley, Meyers et al., 2008) have identified that the minimum clinically important difference in the score for each activity is about 0.5 while 1.5 and 2.0 would identify moderate and large positive changes and negative values would indicate decline. Individuals rated themselves at the beginning of the programme and again at the end.

Results to date We have only completed 10 cases so far. Of these, one person had a large improvement in dyspnoea, one a moderate improvement and two a small improvement; one person deteriorated. For fatigue, three reported a large improvement, one moderate and three a small improvement; no-one deteriorated. For emotional function, there was one large improvement, two moderate and one small and one person deteriorated. For mastery, one person had a large improvement, one a moderate improvement and one a small improvement but five deteriorated.

Conclusion This is only a small sample so far but the evaluation continues. The improvement in dyspnoea and fatigue is encouraging and we hope that over time there will be more improvement in mastery.

\section{P-114 SINGLE NURSE ADMINISTRATION (SNA) CONTROLLED DRUGS - A PHASED APPROACH}

Tracey Taylor, Kate Nolan. Marie Curie, Liverpool, UK
Background Controlled drugs (CDs) are used extensively in end of life care and need to be administered in a timely way. Two staff nurses to check and administer CDs has implications on time, autonomy and person-centred care.

Aims To empower staff to work autonomously in the administration of oral CDs, reduce CD incidents, free nursing time to care and prevent delay in the administration of medication.

Methods SNA would be considered a significant change to process therefore the first step was to introduce this proposed change as part of staff's annual medications training. This consisted of revisiting;

- policy and procedure for CDs

- mapping the process using a medications incident to demonstrate the risk of a two- nurse process.

Root Cause Analysis and human factors were considered. A phased approach was utilised to role out this innovative change over a six months period. Phase 1 consisted of Band 6 staff and nurses that had volunteered to participate. Phase 2 were staff who were initially reserved regarding the SNA but encouraged by the initial success of Phase 1 . Phase 3 took longer as this was a fluid group of staff who were new and lacked experience, had confidence issues due to a previous drug error or needed additional support due to concerns.

Results SNA of CDs showed a reduction in incidents from $72.7 \%$ with two nurses administering to $28.5 \%$ with SNA. Staff reported more autonomy, release of time to care and speed of reaction to patient need.

Conclusion SNA demonstrated a positive impact on personcentred care for all.

\section{P-115 IMPLEMENTING SINGLE NURSE CONTROLLED DRUG ADMINISTRATION. A PRACTICE DEVELOPMENT APPROACH}

Kim Donaldson, Irene Barclay, Dorothy McArthur, Vicky Hill, Richard McKay, Patricia McLaughlin, Lise Brown, Mandy Reid, Kevin Byrne, Kate Crichton, Anna Lloyd, Dot Partington. St Columba's Hospice, Edinburgh, UK

10.1136/bmjspcare-2018-hospiceabs.140

Background In a hospice inpatient unit (IPU), registered nurses (RNs) raised concerns about time taken to administer controlled drugs impacting on patient waiting time for pain and symptom control and limiting the time available for other RN activities.

Aim To enhance person-centred practice around pain and symptom control by using a practice development (PD) approach to implement single nurse controlled drug administration (SNCDA) in a hospice IPU.

Method A Practice Development approach using collaboration, inclusion and participation (McCormack, Manley \& Titchen, 2013) was used throughout.

Preparatory phase

- A literature review and staff engagement sessions to elicit an overview of values and beliefs informed policy, procedure and risk assessments.

- A programme of education was developed in collaboration with the MDT including facilitated critical reflection. 